\title{
Aprendizaje de la matemática en primer año de Ingeniería Agroforestal, I semestre 2019
}

\author{
Learning mathematics in Agroforestry Engineering freshmen students, I semester 2019
}

Javier Osmar Artola García'

\section{Resumen}

Esta investigación analiza el aprendizaje de la matemática en estudiantes de primer año de Ingeniería Agroforestal, para el análisis los indicadores fueron: sexo, edad, etnia, estado civil, situación laboral, número de hijos, tiempo semanal para estudiar matemática, cantidad de consultas semanales hechas al docente de la asignatura y calificación de una serie de estrategias relacionadas con el aprendizaje.

La investigación fue cuantitativa, se aplican técnicas estadísticas para organizar, representar datos y hacer resúmenes estadísticos de las variables del estudio, el alcance es correlacional, porque, se cuantifica la relación entre la actitud hacia la matemática y el rendimiento en la misma.

También valora la actitud hacia el estudio de la matemática, identificando primero la actitud media de cada estudiante, posteriormente se calcula la actitud promedio colectiva. Para hacer un análisis más exhaustivo se comparó la actitud y la calificación por sexo, al final se correlacionó la actitud media con la calificación de la primera evaluación parcial.

Palabras clave: Matemática; actitudes hacia el aprendizaje de la matemática; aprendizaje de la matemática; correlación entre actitud y calificación.

\section{Abstract}

This research analyzes the learning of mathematics in freshmen students of Agroforestry Engineering, for the learning analysis there were taken into account the indicators such as sex, age, ethnicity, marital status, employment status, number of children, weekly time to study mathematics, number of weekly consultations made to the teacher of the subject and qualification of a series of strategies related to learning.

The research has a quantitative approach, since statistical techniques are applied to organize, represent data and make statistical summaries of the study variables, the scope is correlational, because of the relationship between the attitude towards mathematics and academic performance in the class.

It also assesses the attitude towards the study of mathematics, first identifying the average attitude of each student, then the collective average attitude is calculated. To make a more exhaustive analysis, the attitude and the rating by gender were compared, in the end the mean attitude was correlated with the rating of the first partial evaluation.

\footnotetext{
1 Máster en Matemática Computacional, Docente Tiempo Completo del Área de Ciencia Tecnología y Medio Ambiente, URACCAN-Recinto Las Minas, correo: osmartola@gmail.com, https://orcid.org/0000-0001-7047-1914
}

Recibido: 05/03/2020 - Aprobado: 29/09/2020 
Keywords: Mathematics; attitudes towards learning Mathematics; Mathematics learning; attitude and score correlation.

\section{Introducción}

El presente trabajo se desarrolló en la Universidad de las Regiones Autónomas de la Costa Caribe Nicaragüense (URACCAN), Recinto Las Minas, ubicado en el sector número tres del barrio "Pedro Joaquín Chamorro" de la ciudad de Siuna, Región autónoma del Caribe Norte. El propósito general consistió en analizar el aprendizaje de la matemática en primer año de Ingeniería Agroforestal. Entre los objetivos específicos figuran valorar la actitud hacia el estudio de la matemática, identificar las estrategias usadas durante el aprendizaje de la asignatura y por último, correlacionar la actitud y la calificación de la primera evaluación parcial.

El grupo seleccionado estuvo conformado por 26 mujeres y 33 varones para un total de 59 estudiantes que cursan el primer año de la carrera Ingeniería Agroforestal. Estadísticamente el grupo es manejable, por tanto, fue imprescindible la selección de muestra. La investigación es de enfoque cuantitativo, ya que, se aplican técnicas estadísticas para organizar, representar datos y hacer resúmenes estadísticos de las variables del estudio, el alcance es correlacional, porque, se cuantifica la relación entre la actitud hacia la matemática y el rendimiento en la misma.

Entre las estrategias de estudio no se consideran las consultas a otros docentes ni al mismo docente de la asignatura, ya que, se apoyan con sus equipos de trabajo, sólo el $13 \%$ ha abordado al docente para aclarar dudas. Entre los medios para obtener información están las explicaciones recibidas en clase, los apuntes del cuaderno, información de Internet, la plataforma virtual a la que entran para descargar el plan de clase y las guías de trabajo. Para resolver las guías de trabajos se apoyan en aplicaciones instaladas en el teléfono celular, las que usan con más frecuencia son las que permiten una fotografía como entrada de datos y muestran la solución paso a paso.

En los resultados de esta investigación cuantitativa se encontró que al 10.17\% les gusta en realidad las matemáticas; sin embargo, el 93.23\% están entre totalmente de acuerdo y de acuerdo con desarrollar sus habilidades matemáticas. Por otro lado, el $76.27 \%$ están totalmente de acuerdo con que las matemáticas son necesarias y de mucho valor, además el $83.05 \%$ piensa que una preparación fuerte en matemáticas puede ayudarles en su vida profesional.

La actitud promedio hacia la matemática en general obtuvo una puntuación de 80.5, la que se ha tipificado como favorable, es decir, este grupo tiene actitud para enfrentar la matemática en sus estudios universitarios.

El coeficiente de correlaciónline $(r=0.19)$ al entre la actitud hacia la matemática y la calificación obtenida en la primera evaluación parcial es bajo, indicando que no hay relación entre ambas variables, además, se ha deducido que quién no tiene actitud favorable ha obtenido una nota superior al que tiene actitud favorable, es decir, el estudiantado con actitud favorable no ha alcanzado la nota justa para este nivel de actitud.

\section{Revisión de literatura}

En todos los estudios las actitudes hacia las matemáticas se evalúan en base a una adaptación y ampliación realiza por da Silva (2005) del Inventario de Actitudes hacia las Matemáticas (IAM) elaborado por Fennema y Sherman (1976).

Existen varios estudios en los que se correlaciona las actitudes y el rendimiento académico en la asignatura de matemática, tanto para educación primaria, secundaria y universitaria, como el desarrollado por Díaz, Ramírez y Díaz (2016).

Reyes, Porro y Pivorani (2014) en su estudio presentan un análisis del rendimiento académico en el que usan la puntuación media de las actitudes y la nota obtenida. 


\section{Materiales y métodos}

El presente estudio se realizó con los estudiantes de primer año de la carrera Ingeniería Agroforestal que oferta la URACCAN, en el Recinto las Minas, durante el primer semestre del año académico 2019.

Es una investigación con enfoque cuantitativo ya que, se usaron técnicas estadísticas para organizar y representar los datos, además de calcular resúmenes estadísticos de las variables del estudio, el alcance es correlacional, porque, se cuantifica la relación entre la actitud hacia la matemática y el rendimiento en la misma.

La información fue recolectada por el docente de matemática básica en el horario correspondiente a la asignatura. El docente explicó al estudiantado la importancia de la honestidad al momento de llenar el cuestionario. Participaron 26 mujeres y 33 varones para un total de 59 estudiantes, por lo que no fue preciso seleccionar muestra.

La información relacionada con la actitud hacia la matemática se recolectó con una adaptación a las características del grupo de los ítems del Inventario de Actitudes hacia las Matemáticas (IAM) que, consisten en una serie de afirmaciones que usan la escala Likert para calificarlas. Las estrategias se identifican mediante una serie de afirmaciones relacionadas con el aprendizaje, en base a una escala tipo Likert el estudiante califica las que usa.

La calificación de la primera evaluación parcial se obtuvo con las evaluaciones sumativas y formativas propias de la asignatura. Fue indispensable el uso de la computadora para la redacción del informe. El procesamiento de datos se hizo en la versión 3.6.1 del software R, bajo la interfaz gráfica de RStudio 1.2.1335.

\section{Resultados y discusión}

La carrera de primer año de Ingeniería Agroforestal está conformada por $44.07 \%$ del sexo femenino y el 55.93\% del sexo masculino, $66.10 \%$ de los estudiantes tienen a lo sumo 18 años de edad, lo que indica que aún no han madurado lo suficientemente y posiblemente tengan problemas para ubicarse en el nivel universitario.

La etnia predominante es la mestiza con una frecuencia de $98.31 \%$, seguida por la etnia creol con $1.69 \%$. El 91.53\% de los estudiantes son solteros, $1.69 \%$ casados y 6.78\% viven en unión de hecho estable. El 3.29\% de las estudiantes son mujeres que tienen exactamente un hijo. En la situación laboral se encontró que el $66.10 \%$ no trabaja, $3.39 \%$ trabaja permanentemente, $5.08 \%$ trabaja por cuenta propia y $25.42 \%$ trabaja de manera temporal, por tanto, se deduce que este grupo dispone tiempo para dedicar a sus estudios.

El $76.27 \%$ está totalmente de acuerdo con que las matemáticas son una materia necesaria y de mucho valor, $20.34 \%$ está de acuerdo y 3.39\% no está ni de acuerdo ni en desacuerdo con la afirmación anterior. Con respecto a si les gusta realmente la matemática, $10.17 \%$ está totalmente de acuerdo, 37.29\% está de acuerdo, $32.20 \%$ no está ni de acuerdo ni en desacuerdo, $13.56 \%$ está en desacuerdo y $6.78 \%$ está totalmente en desacuerdo.

El 50.86\% están totalmente de acuerdo con desarrollar habilidades matemáticas, 42.37\% está de acuerdo, $5.08 \%$ no está ni de acuerdo ni en desacuerdo y $1.69 \%$ de éstos opina estar en desacuerdo.

El 83.05\% está totalmente de acuerdo con que una fuerte preparación matemática puede ayudarles en su vida profesional, $13.56 \%$ está de acuerdo, $1.51 \%$ no está ni de acuerdo ni en desacuerdo y $1.69 \%$ expresa estar en total desacuerdo con esta afirmación.

El tiempo de estudio se $2.8 \leq t \leq 6.0$ estimó mediante un intervalo al $95 \%$ de confianza obteniendo como resultado donde $t$ es el tiempo en horas por semana. Las principales estrategias para estudiar la asignatura y la puntuación obtenida en cada una se resumen en la Tabla 1. 
Tabla 1: Principales estrategias de aprendizaje

\begin{tabular}{|l|l|l|l|l|l|}
\hline \multicolumn{1}{|c|}{ Estrategias de aprendizaje } & \multicolumn{1}{c|}{ Nunca } & \multicolumn{1}{c|}{ Raras veces } & \multicolumn{1}{c|}{ A veces } & \multicolumn{1}{c|}{ Frecuentemente } & \multicolumn{1}{c|}{ Siempre } \\
\hline Explicaciones del docente & $6.78 \%$ & $3.39 \%$ & $35.59 \%$ & $15.25 \%$ & $38.99 \%$ \\
\hline Apuntes del cuaderno & $0 \%$ & $8.47 \%$ & $22.03 \%$ & $28.81 \%$ & $40.69 \%$ \\
\hline Información en Internet & $6.78 \%$ & $1.69 \%$ & $30.51 \%$ & $22.04 \%$ & $38.98 \%$ \\
\hline Trabajo en equipo & $5.08 \%$ & $5.08 \%$ & $20.34 \%$ & $32.20 \%$ & $37.29 \%$ \\
\hline Software en el celular & $25.42 \%$ & $16.95 \%$ & $27.12 \%$ & $6.78 \%$ & $23.73 \%$ \\
\hline Plataforma virtual & $23.73 \%$ & $3.39 \%$ & $30.51 \%$ & $10.17 \%$ & $32.20 \%$ \\
\hline
\end{tabular}

En la quinta fila de la Tabla 1 se aprecia que el $23.73 \%$ de los estudiantes siempre usa aplicaciones matemáticas en el teléfono celular, entre las principales aplicaciones están las mostradas en la Figura 1.

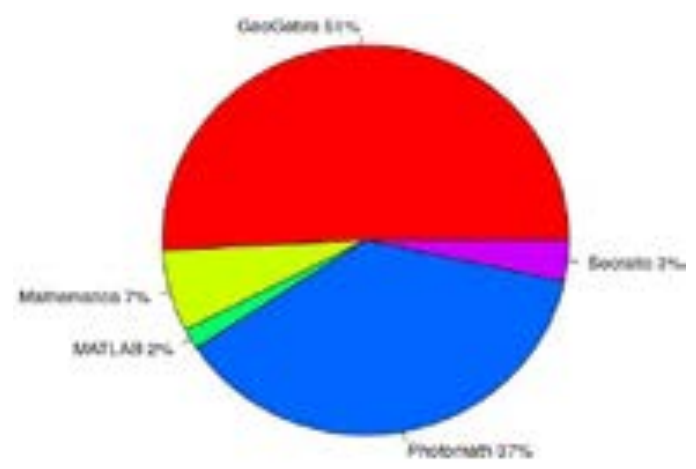

Figura 1: Software usados para estudiar matemática

Entre las aplicaciones mejor valoradas están Photomath y Socratic, puesto que no necesitan introducir parámetros de entrada, basta con tomar una foto al ejercicio y dan como salida la solución, además, disponen de una opción que muestra los pasos usados para generar la respuesta.

El 77.27\% nunca usa computadora para buscar información, leer libros, revisar las guías y la plataforma virtual, por tanto, el teléfono celular es la principal herramienta tecnológica; $61.02 \%$ usa entre siempre y frecuentemente este dispositivo para leer las guías de ejercicios y revisar la plataforma virtual.

Con los ítems de la encuesta relacionados con la actitud se ha calculado la actitud promedio de cada estudiante mediante la puntuación total en la escala (suma de los valores alcanzados en cada ítem) dividida por el número total de afirmaciones.

La escala para evaluar la actitud hacia las matemáticas contempló cinco valores, de esto, la puntuación mínima posible es 23 y la máxima 115, puesto que hay 23 afirmaciones. La actitud promedio en general obtuvo una puntuación de 3.5 en la escala de 1 a 5 y de 80.5 en la escala entre 23 y 115, por tanto, se valora como favorable.

La actitud en los varones es más simétrica o menos dispersa (más homogénea) que, en las mujeres, es decir, la actitud varía más en las mujeres que en los varones. La mediana de la actitud en el sexo femenino es mayor que en el masculino. La distribución de la actitud en el sexo femenino presenta un sesgo a la izquierda, lo que influye sobre la media ubicándola la izquierda de la mediana (Figura 2). 


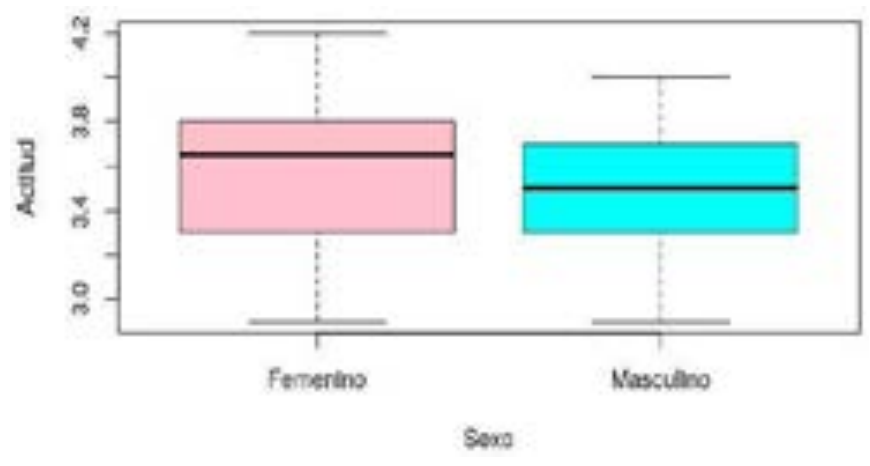

Figura 2: Actitud vs sexo

No hay diferencias significativas en la calificación entre varones y mujeres; sin embargo, las mujeres han obtenido un rendimiento promedio ligeramente mayor en la primera evaluación parcial que el obtenido por los varones, en la Tabla 2 se presenta un resumen estadístico de la calificación por sexo.

Tabla 2: Calificación por sexo

\begin{tabular}{|c|c|c|l|l|l|l|}
\hline Sexo & \multicolumn{1}{|c|}{ Mín } & \multicolumn{1}{|c|}{$\mathbf{Q 1}_{1}$} & \multicolumn{1}{|c|}{ Mediana } & \multicolumn{1}{c|}{ Media } & \multicolumn{1}{c|}{$\mathbf{Q 3}_{3}$} & \multicolumn{1}{c|}{ Máx } \\
\hline Femenino & 35.00 & 44.25 & 53.00 & 53.92 & 64.00 & 71.00 \\
\hline Masculino & 31.00 & 44.00 & 50.00 & 51.73 & 61.00 & 70.00 \\
\hline
\end{tabular}

La ligera diferencia en la calificación se debe a que las mujeres mostraron más responsabilidad que los varones en las distintas evaluaciones, a pesar que 3.29\% tiene un hijo y divide su tiempo entre atender a su hijo, trabajar y estudiar.

Se aplicó el test Shapiro-Wilk para verificar que las variables actitud y calificación siguen una distribución normal, obteniendo $\mathrm{W}=0.96425$ para la actitud y $\mathrm{W}=0.95655$ para la calificación, es decir, ambas variables se distribuyen normalmente, por tanto, Pearson es la prueba adecuada para los datos.

La Figura 3 muestra el diagrama de dispersión de la actitud promedio comparada con la calificación de cada estudiante, la nube de puntos está muy dispersa, lo que significa que existe poca o ninguna relación lineal entre las variables actitud y calificación.

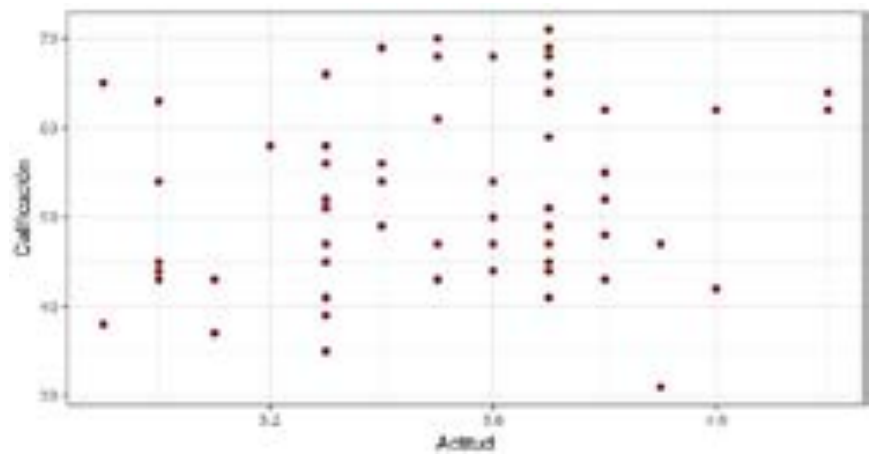

Figura 3: Diagrama de dispersión actitud vs nota

La prueba de correlación entre las variables Actitud y Calificación dio como resultado un valor aproximado para el coeficiente de correlación lineal $r$ de Pearson $r=0.19$, con un intervalo [0.07, 0.42] al 95\% de confianza, los límites del intervalo contienen al cero, este intervalo de confianza sugiere que no existe correlación lineal entre ambas variables. 


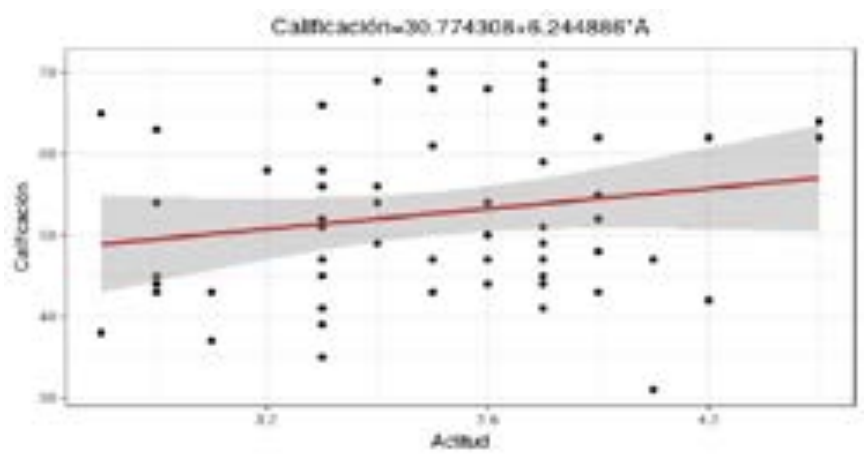

Figura 4: Gráfico del modelo ajustado

El resultado anterior se puede visualizar en la Figura 4 que representa la gráfica del modelo lineal ajustado. El modelo calculado está dado por la ecuación $\mathrm{C}=30.77+6.42 \mathrm{~A}$, donde $\mathrm{C}$ es la calificación y A la actitud.

Para asegurar la no correlación entre ambas variables se aplicará un método no paramétrico, ya que, la variable Actitud es categórica, mediante la prueba Kruskall-Wallis se obtiene: Kruskal - Wallis chi - squared $=12.813, \mathrm{df}=12, \mathrm{p}-$ value $=0.3828$, el valor $\mathrm{p}=0.38>0.05$ , por tanto, se concluye con un nivel de significancia del $5 \%$ que no hay diferencias significativas entre la calificación y la actitud de este grupo de estudiantes, es decir, la calificación no depende de la actitud del estudiantado.

La falta correlación lineal se justifica mediante el siguiente análisis: quién no tiene actitud favorable ha obtenido una nota superior a los que tienen actitud favorable, es decir, el estudiantado con actitud favorable no ha alcanzado la nota justa para su nivel de actitud.

Algunos estudiantes con actitud favorable no mostraron responsabilidad en el desarrollo de actividades orientadas en clase, esto coincide con que el $13 \%$ de los estudiantes ha buscado al docente para consultar, además, han mostrado mucha confianza en las evaluaciones ateniéndose que entienden y que pueden obtener una buena calificación en la próxima oportunidad, por otro lado, el tiempo que están dedicando para estudiar es muy poco.

El factor edad juega un papel importante, al inicio se describió que el 66.10\% tienen a lo sumo 18 años, es probable que aún no se han acomodado a la vida universitaria.

\section{Conclusiones y recomendaciones}

La actitud hacia la matemática se valora como favorable, se reconoce que la matemática es una asignatura necesaria y de mucho valor, que una fuerte preparación matemática podría ayudarles en su vida profesional y desean desarrollar sus habilidades matemáticas.

No se considera diversidad de estrategias para el estudio de la matemática, sólo usan: explicaciones del docente, apuntes del cuaderno, información concreta de Internet, estudio en equipo, información disponible en la plataforma virtual y trabajo con aplicaciones en el teléfono celular.

El tiempo para estudiar matemática no es óptimo, se estima que estudian entre 2.8 y 6 horas semanales revisando los apuntes del cuaderno los días en que les corresponde recibir la clase o realizan trabajos.

No hay correlación entre la actitud y la nota, los estudiantes no han alcanzado la nota acorde a su nivel de actitud. 
Los sistemas de evaluación deben considerar evaluaciones individuales para que la nota sea más justa con respecto al nivel de actitud y desempeño personal de cada estudiante.

\section{Agradecimiento}

Esta publicación obtuvo el financiamiento de: El Fondo de Asistencia Internacionalde los Estudiantes y Académicos Noruegos (SAIH).

\section{Lista de referencias}

da Silva Elza, H. (2005). Actitudes hacia el aprendizaje de las matemáticas (Tesis inéditade doctorado). Universidad de Oviedo, Oviedo, España.

Díaz, N., E., Ramírez, W., D. \& Díaz, M., J., L. (2016). Correlación de las actitudes y el rendimiento académico en la asignatura de matemáticas. Programa de Matemáticas, 3(1). Recuperado de investigaciones.uniatlantico.edu.co/revistas/index.php/MATUA/articleview1511/1224

Fennema, E. \& Sherman, J., A. (1976). Mathematics Attitudes Scales: Instruments Designed to Measure Attitudes toward the Learning of Mathematics by Females and Males. Research in Mathematics Education, 7(5). Recuperado de http://www.jstor.org/stable/748467

Reyes, M., S., Porro, S. \& Pivorani, M., E. (2014). Actitudes y rendimiento académico: su evolución desde química general e inorgánica hasta química orgánica. Colombiana de Química, 43(1). Recuperado de http://www.scielo.org.co/pdf/rcq/v43n1/v43n1ao6.pdf 